\title{
Análisis cualitativo de la educación física escolar en España y Brasil: realidades, similitudes, diferencias y propuestas de mejora \\ Qualitative analysis of school physical education in Spain and Brazil: realities, similarities, differences and proposals for improvement \\ *José Francisco López-Gil, **Emilia Devantel Hércules, **Fernando Renato Cavichiolli *Universidad de Murcia (España), **Universidade Federal do Paraná (Brasil)
}

\begin{abstract}
Resumen. La educación física escolar es una didáctica específica que atañe, tanto a niños como a adolescentes, en la práctica regular de actividad física; sin embargo, resulta llamativo que, a pesar de sus innumerables beneficios, su prestación se esté viendo mermada en numerosos países del mundo. Por ello, resulta necesario que los gobiernos tomen medidas en los currículos escolares. A partir de esta necesidad, emana el interés por conocer la situación actual de la educación física escolar desde distintos lugares del mundo; siendo utilizados para tal fin dos países muy diferentes, tanto en su localización geográfica como en su cultura, como son España y Brasil. Así, aparecen ítems referentes a legislación, horas lectivas, enseñanza, formación docente y actividades deportivas complementarias, en relación con la educación física. El objetivo de este artículo fue la comparación descriptiva y cualitativa de las realidades, similitudes y diferencias entre estos países, así como la exposición de propuestas de mejora aplicables; destacando la importancia de elevar el número de horas lectivas de educación física escolar.
\end{abstract}

Palabras clave. Educación Física; Educación Primaria, Maestros; Evaluación de la Educación; Enseñanza; Educación en Salud.

\begin{abstract}
School Physical education is a specific didactic that involves both children and adolescents in regular practice of physical activity; however, it is striking that, despite its innumerable benefits, its provision is being undermined in many countries in the world. It is therefore necessary for governments to act in school curricula. From this need comes the interest to know the current situation of school physical education from different places in the world; being used for this purpose two very different countries, as much in their geographical location as in their culture, as Spain and Brazil. Thus, there are items referring to legislation, teaching hours, teaching, teacher training and complementary sports activities in relation to physical education. The aim of this article was the descriptive and qualitative comparison of the realities, similarities and differences between these countries, as well as the exposition of applicable improvement proposals; highlighting the importance of increasing the number of school hours of physical education.
\end{abstract}

Keywords. Physical Education; Primary Education; School teachers; Educational Measurement; Teaching; Health Education.

\section{Introducción}

La educación física (EF) conforma una disciplina que inmiscuye a los jóvenes, tanto a niños como a adolescentes, en la práctica regular de actividad física (AF); sin embargo, en estas últimas edades se contemplan elevadas tasas de abandono de la práctica regular de la misma (Franco et al., 2017). Este hecho, ha suscitado en los últimos años el interés de las investigaciones científicas, en torno a la EF y su inexorable promoción de la práctica de AF (Sallis et al., 2012; Taylor, Spray \& Pearson, 2014).

Por otra parte, aunque la Organización Mundial de la Salud (OMS) ha catalogado como pandemia los elevados niveles de sedentarismo, así como el riesgo potencial asociado de diversos tipos de enfermedades no transmisibles, como la obesidad, los accidentes cerebrovasculares, el cáncer, la diabetes, las enfermedades respiratorias crónicas o las enfermedades cardíacas, llama la atención que la prestación de la enseñanza de EF se esté viendo reducida en todos los lugares del mundo, aumentando estas graves consecuencias de forma exponencial. Por ello, es necesario que los gobiernos lleven a cabo acciones en los currículos escolares de todo el mundo (Organización de las Naciones Unidas para la Educación, la Ciencia y la Cultura, [UNESCO], 2015).

A partir de esta necesidad, y postulándose la escuela como un escenario que permite discusiones para cuestiones relacionadas con la AF y la salud, se justifica el interés por

Fecha recepción: 16-01-19. Fecha de aceptación: 29-04-19

José Francisco López-Gil

josefranciscolopezgil@gmail.com comparar la situación actual de la EF desde distintos lugares del mundo; decantándose, el presente trabajo, por dos países diferentes con culturas muy distintas, como son: España, en Europa y Brasil, en Sudamérica.

Así, en España, el Informe Eurydice de la Comisión Europea, indicó que, el 80\% de los jóvenes españoles, solo realiza AF en el contexto escolar (Eurydice, 2013). Por otra parte, en lo que respecta a Brasil, el estudio realizado por el Ministério do Esporte señaló que el 69,3\% de la población inicia su contacto con el deporte entre las edades de 6 a 14 años, y que, el 40,8\% de los participantes, comienza la práctica deportiva en el ámbito escolar (Ministério do Esporte, [ME], 2013).

Como vemos, estos antecedentes reafirman la importancia de las clases de EF; no sólo por los numerosos efectos que puede tener para la salud que los niños reciban una EF de calidad (OMS, 2018a), sino también para obtener otra serie de beneficios, en relación a: los logros académicos, ya que tanto la EF como otras formas de AF, aumentan la capacidad de atención de los discentes, así como su control y velocidad de procesamiento cognitivo (Ardoy et al., 2014; Coe et al., 2012; Ericsson \& Karlsson, 2014; Haapala, 2013; Torrijos-Niño et al., 2014); la inclusión social, en especial en aquellas poblaciones con elevada vulnerabilidad social (Centro de Estudos e Pesquisas em Educação, Cultura e Ação Comunitária, [CENPEC], 2007; Moll, 2012); así como la alfabetización y la participación activa de las personas en la sociedad actual, a través de la relación entre el movimiento, la comunicación y el aprendizaje de valores (UNESCO, 2015).

En cuanto a la metodología de este trabajo, se abogó por un enfoque cualitativo y descriptivo, haciendo uso de docu- 
mentos legislativos y de referencia, en cuanto a políticas públicas se refiere, para localizar las similitudes y diferencias entre Brasil y España, así como las propuestas de mejora para ambos países.

El objetivo de este artículo fue analizar aspectos relevantes de la EF escolar en Brasil y España, para llevar a cabo discusiones sobre aproximaciones y distanciamientos posibles. Para ello, se seleccionaron diversas categorías de manera intencional, puesto que, al parecer de los autores, estos ítems resultan de mayor relevancia para facilitar la comprensión de la revisión llevada a cabo. No obstante, estos ítems corresponden a procesos inherentes a las prácticas escolares que tienen relación con la EF; componiéndose a partir de las leyes de cada país y desdoblándose por: legislación, jornada lectiva, enseñanza de la EF, formación del docente de EF y actividades deportivas complementarias.

\section{Análisis de la EF en España}

\section{Perfil de la EF en escuelas públicas de España: a nivel legislativo}

En el contexto educativo español, la EF en edad escolar viene regida por el Real Decreto (RD) 126/2014, de 28 de febrero, que establece el currículo básico de la Educación Primaria (Ministerio de Educación, Cultura y Deporte, [MECD], 2014), y que, a su vez, depende de la Ley Orgánica 8/2013, de 9 de diciembre, para la Mejora de la Calidad Educativa(LOMCE)(MECD, 2013).

En esta última, la EF (junto a religión, valores cívicos y sociales y educación artística) se ha categorizado como una materia específica de todas las etapas educativas. En estas asignaturas, se establece una autonomía más alta por parte de las comunidades autónomas, a la hora de establecer los horarios y los contenidos de las asignaturas, así como a la hora de establecer su oferta académica (MECD, 2013, p.14).

Así, el gobierno español genera un contexto curricular general y flexible para la EF, de forma que pueda ser modificado, de manera posterior, por las comunidades autónomas, así como por las propias escuelas (Méndez, Fernández-Río, Méndez \& Prieto, 2015; Méndez, Pérez, Méndez, Fernández \& Prieto, 2017).

No obstante, este panorama educativo, puede concebirse de dos formas distintas; en primer lugar, como una opción de flexibilidad curricular, ya que proporciona mayor capacidad de decisión a las comunidades autónomas, a los centros educativos y a los propios docentes, a la hora de escoger el currículo necesario según el contexto. Por otro lado, como una opción de decisión arriesgada, ya que implica una pérdida de la estabilidad que garantiza el currículo en las asignaturas troncales, para todo el país. Además, en la línea de esta perspectiva anterior, es posible producir planteamientos muy dispares de EF, donde destaca la uniformidad entre las 17 comunidades autónomas del país (de los Mozos \& Touya, 2014).

En otro orden de ideas, tanto la LOMCE, como el RD 126/ 2014, hacen hincapié en la importancia que reside en la EF a la hora de que los discentes adquieran las siete competencias clave (López-Gil, 2018); no obstante, ni la LOMCE ni el RD 126/2014 las abordan, ni tampoco indican la forma en la que la EF debe contribuir a su mejora (Muñoz, 2014). A pesar de este hecho, en la introducción del RD 126/2014, se resalta la importancia de que los alumnos desarrollen la competencia motriz, entendida como un conjunto de conocimientos, habilidades y actitudes relacionadas con la conducta motora (cuerpo, movimiento y relación con el entorno).

\section{Perfil de la EF en escuelas públicas de España: a nivel de jornada lectiva}

La carga lectiva para los discentes es de 20 horas semanales, repartidas en función de la preferencia de los centros. Si bien los centros educativos, pueden determinar la carga horaria correspondiente a las diferentes asignaturas (MECD, 2013, p.14), la mayoría de los mismos agrupa el horario lectivo entre las 9h de la mañana, hasta las $14 \mathrm{~h}$ de la tarde, variando ligeramente en los meses de septiembre (inicio escolar) y junio (final de curso). Por otra parte, cabe destacar que algunos colegios adquieren un horario que incluye un descanso para comer, relevando estas horas a la tarde; no obstante, esta práctica no suele ser muy habitual en las escuelas españolas.

En otro orden de ideas, los niños tienen la opción de realizar la comida del mediodía en el comedor escolar, siempre que abone la cuota económica correspondiente, o disponga de beca para tal fin. El resto de comidas diarias, corren por cuenta del discente (desayuno, almuerzo, merienda, etc.).

\section{Perfil de la EF en escuelas públicas de España: a nivel de enseñanza de $\mathrm{EF}$}

Si bien España presenta disparidad en cuanto al número de horas dedicado a la enseñanza de EF, la mayoría de los centros educativos disponen de dos horas semanales. No obstante, para facilitar su comprensión, hemos reunido las diferentes cargas lectivas para la EF en las distintas comunidades autónomas (Tabla 1).

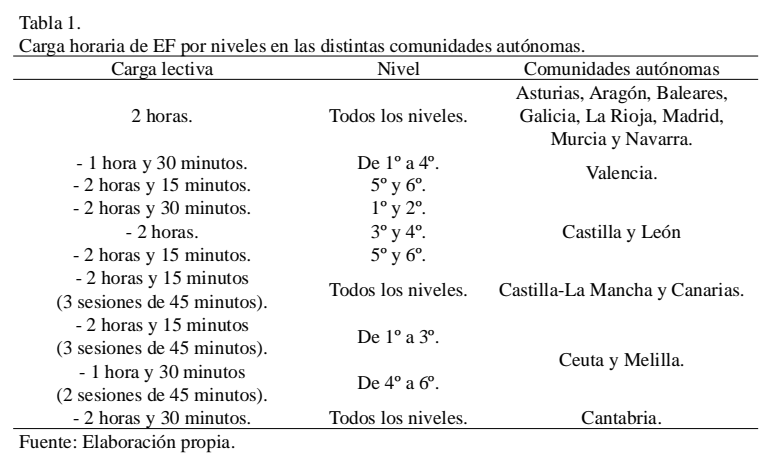

\section{Perfil de la EF en escuelas públicas de España: a nivel de formación docente}

En cuanto a la formación de los maestros de Educación Primaria, la antigua Ley Orgánica de Educación (LOE) (2006) (parcialmente modificada por la LOMCE), en el punto 2 de su artículo 93, señala que la Educación Primaria será impartida por maestros, que tendrán competencia en todas las áreas de este nivel. La enseñanza de la música, de la EF, de los idiomas extranjeros o de aquellas otras enseñanzas que determine el Gobierno, previa consulta de las comunidades autónomas, serán impartidas por maestros con la especialización o cualificación correspondiente (Ministerio de Educación y Ciencia, [MEC], 2006, p. 61). 
Como podemos observar, para ser maestro (profesor) de EF en escuelas públicas españolas, es requisito obtener el Grado en Educación Primaria, con la correspondiente mención en EF. De tal modo, los maestros que adquieran el grado, deberán desempeñar todas las áreas curriculares (ciencias experimentales, ciencias sociales, matemáticas, lenguas, educación musical, plástica y visual) y un cierto grado de especialización en EF (MEC, 2007, p. 53750).

Perfil de la EF en escuelas públicas de España: a nivel de actividades deportivas complementarias

Haciendo referencia a las actividades deportivas complementarias realizadas en las escuelas públicas, la LOE, en el punto 6 de su disposición adicional decimoquinta, indica que las Administraciones educativas deben modelar los procedimientos para la utilización de los centros educativos, por parte de las autoridades municipales, en actividades educativas de tipo cultural, deportivo y/o social, fuera del horario escolar. Asimismo, en el punto 7, señala que las Administraciones educativas, deportivas y municipales, colaborarán para establecer procedimientos que hagan posible la utilización de las instalaciones deportivas para los centros docentes o para los municipios. (MEC, 2006, pp. 92-93).

\section{Análisis de la EF en Brasil}

\section{Perfil de la EF en escuelas públicas de Brasil: a nivel legislativo}

En Brasil, la EF aparece como un componente curricular a través de la Lei de Diretrizes e Bases da Educação (LDB) 9394/96, teniendo repercusión en las orientaciones y políticas públicas, posteriormente establecidas por el Gobierno brasileño. Así, en su artículo 26, se manifiesta la obligatoriedad de la EF, como un componente curricular de la educación básica (Ministerio da Educação, [MEC], 1996).

La LDB constituye y orienta el sistema nacional de educación brasileño, el cual presenta unas políticas públicas amplias, considerando la realidad social del país. Asimismo, las directrices curriculares buscan atender las generalidades del país, evidenciando las diferentes posibilidades para la ampliación de perspectivas en las diferentes regiones (MEC, 1996).

Por otra parte, Saviani (2010) señala que, aunque la LDB regula las acciones del sistema educativo, delega en la autonomía estatal y municipal las decisiones curriculares, en función de la cultura local y sus preferencias; posibilitando así, el mantenimiento, el respeto y el fortalecimiento de la diversidad brasileña.

En línea de lo anterior, las primeras acciones efectivas se describen en los Parámetros Curriculares Nacionais (PCN) (MEC, 1997) y se configuran como una propuesta flexible, abierta y carente de imponer qué contenidos han de ser trabajados. Además, se hace énfasis en otras temáticas consideradas importantes como salud, medio ambiente, calidad de vida, género, entre otras.

Posteriormente, en el año 2010, fueron creadas las Diretrizes Curriculares Nacionais para a Educação Bási$c a$, que retoman los principios de enseñanza de calidad, el aprendizaje efectivo del estudiante o la participación colectiva de la comunidad en la gestión democrática escolar, entre otros (MEC, 2010). Igualmente, este documento propone líneas generales e indica las bases para la elaboración colectiva de la Base Nacional Comum Curricular (BNCC); documento que fue lanzado en 2017 y que, actualmente, está en proceso de implantación en Brasil.

Por otro lado, la BNCC persigue establecer objetivos de aprendizaje para todos los estudiantes brasileños de la educación básica, con lo que se pretende delimitar el currículo mínimo; con contenidos, clasificaciones, metodologías y principios basados en aprendizajes por competencias específicas. Además, otorga libertad para la ampliación curricular, siendo esa función inherente a los estados y municipios (MEC, 2017).

La EF está incluida en la BNCC junto al área de lenguajes, debido a que la misma, trabaja en el desarrollo de prácticas corporales codificadas culturalmente e insertadas a nivel social. En ella, se incluyen conocimientos corporales de tratamiento y enseñanza lúdica, que se desarrollan pedagógicamente; generando experiencias y participaciones de los estudiantes durante la vida escolar y promoviendo, así, posibilidades para una vida sana y activa (MEC, 2017,p. 211).

Además, el documento resalta que las distintas perspectivas de la EF, giran en torno a tres elementos: el movimiento corporal como elemento esencial; la organización interna (de mayor o menor grado) pautada por una lógica específica; y el producto cultural vinculado con el ocio/entretenimiento y/o cuidado del cuerpo y la salud (MEC, 2017, 211).

No obstante, la concepción apuntada por los PCN y la BNCC es la referente a la cultura corporal, englobando aspectos socioculturales, fisiológicos y técnicos que componen la EF. Se parte del concepto de que el cuerpo y sus movimientos adquieren significados cuando se insertan en un contexto específico. A partir de ese concepto, la EF está pautada en cinco ejes de contenidos centrales: juegos, danzas, gimnasia, luchas y deportes, que se abordan siguiendo principios de desarrollo crítico y social (MEC, 1997).

\section{Perfil de la EF en escuelas públicas de Brasil: a nivel de jornada lectiva}

Las escuelas brasileñas, en su mayoría, se organizan en turnos de 20 horas semanales para los estudiantes de Ensino Fundamental. El funcionamiento de las clases gira en torno a las 4 horas, realizándose en turnos de mañana, tarde o noche. Así, la EF se encuentra en esta organización de forma variable.

Por otra parte, algunos autores como Bracht (2010), hacen referencia a que la disciplina tiene muchos desafíos. Por un lado, la profesionalización de los profesores de los primeros años de Ensino Fundamental; por otro, la definición del tiempo de desarrollo de la clase y temporalidad semanal, ya que puede ser de dos o tres veces a la semana. Así, la EF se encuentra frágilmente establecida en el espacio escolar, al contrario que otros componentes curriculares, y siendo, por tanto, más susceptible a improvisaciones cotidianas.

Perfil de la EF en escuelas públicas de Brasil: a nivel de enseñanza de $\mathrm{EF}$

En los últimos 10 años, han existido controversias sobre la disminución del tiempo para el desarrollo de la EF escolar, 
apareciendo propuestas para modificar las leyes y establecer las dos horas semanales; independientemente de la temporalidad destinada a las clases. Sin embargo, estas proposiciones no llegaron a ser tramitadas, prevaleciendo lo establecido en la LDB. Así, la EF aparece como una disciplina obligatoria y que debe ser realizada en, por lo menos, dos horas semanales. Asimismo, y con independencia de cómo se dividan las clases (40, 50 o 60 minutos), deben ser completados los 120 minutos obligatorios por semana.

\section{Perfil de la EF en escuelas públicas de Brasil: a nivel de formación docente}

En Brasil, los profesionales de EF realizan su formación mediante la graduación en bacharel y la graduación en licenciatura; apareciendo esta división a partir del año 2000, en un intento de formar profesionales más capacitados para ambas áreas.

En esta línea, el bacharelado está orientado hacia un área no formal, desarrollando un perfil para salas de musculación, deportes, ocio o fitness, entre otros. Por el contrario, la licenciatura en EF desarrolla el trabajo para la actuación escolar, con independencia de las edades.

Perfil de la EF en escuelas públicas de Brasil: a nivel de actividades deportivas complementarias

En Brasil, las actividades deportivas complementarias, quedan sujetas a la ampliación de la jornada escolar; siendo ésta, prolongada hasta siete y ocho horas de actividades escolares.

Así, cuatro de ellas se destinan para el desarrollo de prácticas dirigidas a actividades relacionadas con el arte, la ciencia, la educación ambiental y el ocio, así como ciertas actividades deportivas complementarias (MEC, 2013).

\section{Comparación entre EF escolar de España y Brasil}

Durante este proceso de comparación entre sendos países, se utilizaron las siguientes categorías de análisis: legislación, jornada lectiva, enseñanza de la EF, actividades deportivas complementarias y formación del docente de EF.

Perfil de la EF en escuelas públicas: a nivel legislativo

En el contexto de ambos países, existen indicaciones referentes al trabajo de la EF orientado a las competencias motoras, afectivas y sociales, además del abordaje relacionado a la salud y calidad de vida. Aun así, destaca que, Brasil y España, poseen orientaciones parecidas en lo que se refiere al currículo y sus posibilidades relacionadas a la autonomía de los estados/regiones para establecer contenidos relacionados con la práctica de AF.

Además, en este apartado, también resulta remarcable el hecho de que sendos países presenten similitudes a nivel legislativo, a pesar de ostentar un distanciamiento tan pronunciado entre las fechas en las que se establecen esas perspectivas (LDB en 1996 y RD en 2014).

\section{Perfil de la EF en escuelas públicas: a nivel de jornada lectiva}

A pesar de que la carga horaria destinada es de 20 horas semanales, la organización en horas y turnos lectivos es

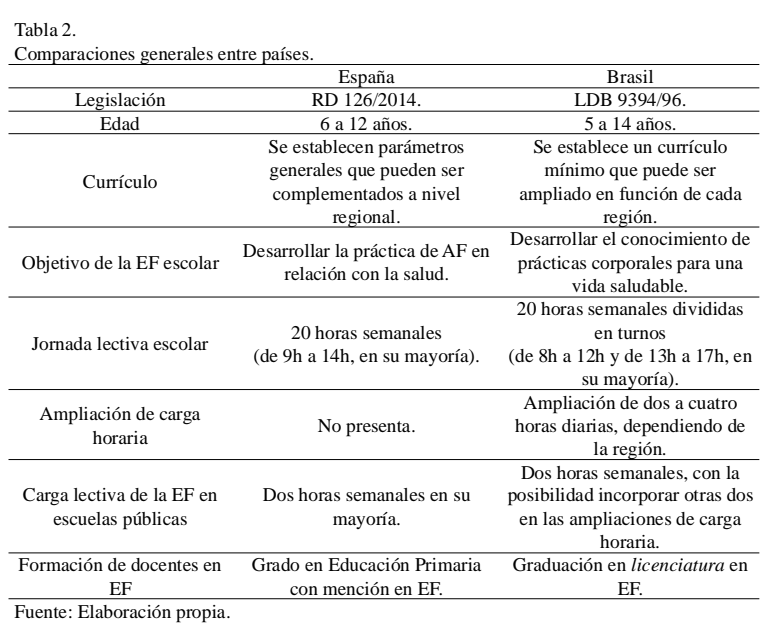

muy diferente (Tabla 2). Principalmente, en lo que se refiere a la temporalización de la carga horaria escolar, se percibe que Brasil se desmarca de España, debido a la alta demanda de atención escolar. Las escuelas brasileñas necesitan realizar atendimientos de cuatro horas diarias en diferentes turnos (mañana y tarde); aproximándose así, a las características de áreas con gran densidad de población, en las que predominan áreas carentes de asentamientos o relaciones poblacionales; produciéndose un desplazamiento de la población hacia áreas con un coste de vida más bajo (CENPEC, 2007). Por el contrario, en España el funcionamiento se produce en un turno único, atendiendo a la población con efectividad.

En otro orden de cosas, la ampliación de la jornada lectiva es un tema en boga, en lo que a Brasil se refiere. Este hecho, queda justificado por la vulnerabilidad social, que exige una mayor demanda de políticas públicas, en relación a la seguridad del ciudadano y al ámbito educativo (Moll, 2012). Por ello, el gobierno federal brasileño presenta interés en la ampliación de las jornadas educativas; colaborando para ello con turnos educativos amplios, y aumentando así, el tiempo en que el estudiante queda bajo la tutela del Estado.

En esta línea, la educación en jornada ampliada pasa a ser una presión constante para abarcar a niños y jóvenes, principalmente en situaciones de riesgo y vulnerabilidad social; es por ello que, los estados y municipios de Brasil presentan diferentes medidas al respecto de esta temática (CENPEC, 2007). Así, desde el año 2007, han sido desarrolladas diversas políticas para aumentar la jornada escolar, en especial, el Programa Mais Educação (MEC, 2013) que fue implantado para logar un mayor desarrollo del arte, el deporte, la tecnología, el portugués y las matemáticas.

Como podemos observar, este atendimiento a los discentes en jornada ampliada es una de las principales diferencias entre Brasil y España.

\section{Perfil de la EF en escuelas públicas: a nivel de ense- ñanza de $\mathrm{EF}$}

Con enfoques metodológicos parecidos, en ambos países la EF busca el desarrollo del sujeto en conjunción con un estilo de vida saludable, a través de principios morales y éticos, y donde destaque la aplicabilidad de las acciones con componente social. La principal discrepancia está en los objetivos generales de la propuesta curricular, ya que, en 
Brasil, el enfoque se centra en el conocimiento de las prácticas corporales, para que esas nociones amplíen las posibilidades de mantenimiento de la práctica de ejercicios y deportes para la vida (Darido \& Rangel, 2005). Así, la EF es concebida como un conjunto de lenguajes, problematizando las vivencias y experiencias corporales y construyendo conocimiento sobre ellas, así como sobre el propio cuerpo (MEC, 1997; 2017). Por el contario, en España, el foco está más orientado al desarrollo de la salud a través de EF, siendo parte del currículo de EF, cuestiones como la alimentación sana y el desarrollo sostenible.

En Brasil, todas las temáticas que pueden contribuir a la construcción y desarrollo del sentido crítico y ciudadano, como la salud, la sexualidad, la ética, el consumo, la sostenibilidad, o el medio ambiente, entre otras, son tratadas como temas transversales; es decir, que atraviesan todas las disciplinas, pero no constan en especificidad en el currículo de EF (MEC, 1997).

Además, se percibe que, en Brasil, hay un esfuerzo en producir un currículo cultural, que sea reflexivo y que produzca significados para el conocimiento y sus experiencias (Nunes \& Neira, 2017). Por ello, las perspectivas metodológicas y las indicaciones curriculares se aproximan mucho más a la expresión de sentimientos, ideas y valores, constituyendo prácticas corporales que se vinculan y están marcadas por relaciones entre clase social, religión, etnia, género, etc.

También sobre la cuestión curricular, en la BNCC se estipula el trabajo mediante las siguientes temáticas: juegos; deportes; gimnasia; bailes; luchas y prácticas corporales de aventura. Estas temáticas son constitutivas del currículo mínimo estipulado para el territorio nacional y para la modalidad de Ensino Fundamental (5 a 14 años). Así, destaca que el deporte es indicado desde edades tempranas, con configuraciones adaptadas a las franjas etarias y con perfil lúdico, al contrario de lo que sucede en España, donde el deporte como tal, es practicado en edades más avanzadas.

\section{Perfil de la EF en escuelas públicas: a nivel de forma- ción docente}

Desde otra perspectiva, es en el contexto de la formación profesional del profesor de EF donde mayores discrepancias podemos encontrar. En España, el docente de EF escolar, debe realizar el grado en Educación Primaria. Esta titulación, está planificada para realizarse en cuatro cursos académicos; dividiéndose en una formación de carácter general de tres cursos y medio y una formación específica, correspondiente a la mención en EF (medio curso académico). Asimismo, en Brasil, el docente de EF debe realizar una Licenciatura en EF, también planificado para cuatro cursos académicos, para poder ejercer en el ámbito escolar.

Como vemos, la formación profesional del docente de EF presenta similitudes entre países, en cuanto a duración. No obstante, los contenidos inmersos en sendas titulaciones varían notablemente. Así, en España, la EF escolar se engloba dentro de las Ciencias Sociales, mientras que, en Brasil, lo hace dentro de las Ciencias de la Salud. Otro aspecto destacado es que, en España, mediante la titulación propicia para ello, el docente puede actuar única y exclusivamente en el ámbito de Educación Primaria; mientras que, en Brasil, ade- más de en Ensino Fundamental, también puede actuar en Ensino Medio (semejante a la Educación Secundaria española), o en otras perspectivas como Educação Infantil e Educação para Jovens e Adultos. En el currículo se describen posibilidades pedagógicas, didácticas y principalmente técnicas y operativas. La formación está destinada al conocimiento de las prácticas y ejercicios y sufre adaptaciones en algunas perspectivas educativas. El currículo de graduación todavía permite que pueda realizarse la especialización de bacharel en EF, así que muchos de los profesionales formados en Brasil consideran la formación en la licenciatura como una posibilidad en su carrera, pero se concentran inicialmente en acciones de trabajo no formales.

Perfil de la EF en escuelas públicas: a nivel de actividades deportivas complementarias

Las condiciones de ampliación de carga horaria permiten a Brasil un amplio abanico de posibilidades de trabajo para las horas dedicadas a actividades deportivas complementarias. En esta ampliación de jornada escolar concurren una diversidad de proposiciones, tales como: proyectos educativos deportivos, ONGs, escuelas con oferta de actividades en contratación y ampliación de carga horaria de la propia unidad escolar, enfocando el aumento de la práctica de la EF escolar en forma de actividades deportivas, ejercicios y prácticas corporales diversas. Estas acciones se constituyen sin costos adicionales, es decir, son ofertas públicas, en espacios escolares y atienden prioritariamente a la población de vulnerabilidad social (CENPEC, 2007).

Lo que difiere en España es que, en las escuelas públicas se pueden desarrollar actividades complementarias, pero hay un costo de mantenimiento y desarrollo. La legislación permite que haya acciones deportivas, pero no son desarrolladas directamente por el centro escolar o las administraciones públicas.

\section{Propuestas de mejora}

Dentro del ámbito legislativo, podemos observar que, a pesar de ser leyes diferentes con sus propias particularidades, tanto en LOMCE y RD 126/2014, como en LDB 9394/96, dejan constancia de la importancia que supone la EF para un desarrollo y crecimiento saludable por parte de los niños.

Este hecho, viene a confirmar la importancia otorgada a la EF como vehículo para la realización de AF, debido a que los beneficios que proporciona para la salud cuentan con un amplio respaldo de la evidencia científica y son inexorables, tanto a un riesgo más bajo de padecer enfermedades cardiovasculares, diabetes, hipertensión y algunos tipos de cáncer, como a efectos beneficiosos sobre la salud mental o al mantenimiento de un peso corporal saludable (International Society for Physical Activity and Health, [ISPAH], 2017; OMS, 2014; Physical Activity Guidelines Advisory Committee, 2018; Sallis et al., 2016; Warburton, Charleswhorth, Ivey, Nettlefold \& Bredin, 2010).

A pesar de ello, nos encontramos con que solamente 20\% de los adolescentes cumplen con las recomendaciones de la OMS de practicar AF de moderada a vigorosa, durante al menos 60 minutos diarios (OMS, 2014). Por ello, poniendo de manifiesto la fuerte asociación entre AF y la prevención 
de las principales enfermedades no transmisibles, la OMS se ha propuesto como objetivo, logar para 2030 una disminución relativa del $15 \%$ en la prevalencia mundial de personas insuficientemente activas, tanto en adultos como adolescentes (OMS, 2018b).

En esta línea, una de las vías para fomentar la práctica de AF en escolares es mediante el ofrecimiento de EF de calidad (OMS, 2018a), ya que tal y como recoge la Carta Internacional de la Educación Física, la Actividad Física y el Deporte, tanto la EF como el deporte, podrían contribuir a proteger y optimizar la salud, soportando de manera más eficaz los inconvenientes propios de la vida moderna (UNESCO, 1978).

Del mismo modo, el Plan de acción de Kazán indica que la EF en el entorno escolar puede generar conocimientos básicos sobre el cuerpo y la salud del mismo; favoreciendo una vida prolongada y saludable, así como previniendo enfermedades no transmisibles mediante un estilo de vida activo(UNESCO, 2017).

Por otro lado, no solamente el hecho de realizar AF presenta beneficios para la salud de los discentes. La EF diaria (aumentando los niveles de AF de manera inequívoca), es una forma viable de mejorar, no únicamente las habilidades motoras, sino también el rendimiento académico (Ericsson \& Karlsson, 2014). De hecho, y tal como recoge el último Informe PISA de 2015, los países que destinan un número mayor de horas a la realización de EF, como Singapur o Finlandia, obtienen las mejores calificaciones a nivel académico, tanto en ciencias, lectura, como en matemáticas (Organización para la Cooperación y el Desarrollo Económicos, [OECD], 2016).

Así, en los últimos años, han sido llevados a cabo numerosos estudios que han demostrado una asociación positiva entre la práctica de AF y el rendimiento académico, tanto en niños (Haapala, 2013; Torrijos-Niño et al., 2014) como en adolescentes (Ardoy et al., 2014; Coe et al., 2012).

En esta línea, algunos estudios han analizado los efectos sobre la realización de un número de horas mayor para EF, como el de Ardoy et al. (2014), que acometieron un estudio de intervención comparando, un grupo con dos horas de EF semanal frente a dos grupos de cuatros horas (con distinta intensidad), concluyendo que, aumentar tanto el número de horas de EF escolar como la intensidad durante las mismas, produce mejoras positivas en el rendimiento cognitivo de los alumnos, así como en el promedio de calificaciones de todas las asignaturas. Asimismo, Ericsson y Karlsson (2014), encontraron resultados positivos, en su estudio de intervención, en las calificaciones académicas de lengua, matemáticas e inglés, en el grupo que tuvo más horas semanales de EF en comparación a los que realizaron un número menor; concluyendo que, a mayor número de horas dedicadas a EF en colegios e institutos, mejores son las habilidades motoras de los discentes, así como su capacidad de aprendizaje.

Al hilo de lo anterior, en una revisión sistemática llevada a cabo por Trudeau y Shephard (2008), se determinó que incluir una hora diaria extra de EF dentro del horario lectivo, puede lograr mejoras en la calificación media de las asignaturas escolares; sin influir de forma negativa al rendimiento académico.

Por consiguiente, y haciendo referencia a todos los factores citados previamente, nos planteamos si, tanto España como Brasil, deberían replantearse ampliar el número de ho- ras establecidas para la asignatura de EF; puesto que, tanto para Educación Primaria como para Ensino Fundamental, la cantidad de sesiones gira en torno a dos por semana, siendo más baja que otros países.

Por otro lado, y en relación a la formación docente del profesional de EF, el hecho de que, en función del país analizado, la EF se encuentre en Ciencias Sociales, Ciencias de la Salud o, incluso, en Ciencias del Deporte, nos advierte del carácter multidisciplinar que la EF posee. Al respecto, resulta difícil posicionarse dentro de un ámbito de estudio u otro, así como hacer recomendaciones de dónde la EF encajaría mejor. No obstante, en el debate de los Comités de Expertos del Consejo-COLEF, realizado recientemente en España, se llegó al consenso en la importancia de no basar únicamente la EF en aspectos fisiológicos; dejando de lado los beneficios cognitivos, emocionales y sociales (Aznar, 2018), como el bienestar general, el disfrute o la satisfacción de los discentes (Águila \& López, 2019). Por ello, resulta una tarea de elevada complejidad indicar el área concreta donde mejor quedaría ubicada la EF, por lo que consideramos que su abordaje debe ser realizado desde distintas perspectivas. No obstante, se requiere que, desde cualquier área que la EF sea abordada, los docentes de EF reciban una formación de calidad, con una carga lectiva óptima; en aras de garantizar que se produzcan en los mismos, una serie de procesos de tipo crítico, reflexivo y constructivo que propicien la mejora de la labor docente (Cañabate, Tesouro, Puiggali \& Zagalaz, 2019); así como el trabajo de la competencia comunicativa desde diversos roles (emisor, receptor, comunicador en el aula, participante en reuniones, tutor, etc.) (Gallego-Ortega \& Rodríguez-Fuentes, 2018).

\section{Comentarios finales}

A pesar de las distinciones culturales y demográficas entre dos países tan diferenciados como España y Brasil, encontramos ciertas aproximaciones en lo que a EF escolar se refiere. No obstante, al mismo tiempo, ciertas diferencias importantes pueden ser observadas.

Tanto en España como en Brasil, los aspectos que rodean a la EF escolar, presentan aspectos positivos, así como susceptibles de mejora; sin embargo, este acercamiento puede ofrecer nuevas ideas de pensamiento, a la hora de tomar de ejemplo algunas de las cuestiones planteadas en este trabajo.

Por último, consideramos prioritario que las políticas públicas sean modificadas en aras de aumentar las horas lectivas de la EF escolar, equiparándose a otros países con un mayor número de horas; puesto que, este hecho, podría generar de manera inequívoca, no sólo beneficios de tipo fisiológico en los escolares, sino también cognitivos, emocionales y/o sociales.

\section{Referencias}

Ardoy, D. N., Fernández-Rodríguez, J. M., Jiménez-Pavón, D., Castillo, R., Ruiz, J. R., \& Ortega, F. B. (2014). Aphysical education trial improves adolescents' cognitive performance and academic achievement: The EDUFIT study. Scandinavian Journal of Medicine \& Science in Sports, 
24(1), 52-61. https://doi.org/10.1111/sms.12093

Aznar, M. (2018). Editorial: La actualidad de la actividad física y el deporte a debate desde los comités de expertos del Consejo-COLEF. Revista Española de Educación Física y Deportes, 420, 15-21. Recuperado de: http:// www.reefd.es/index.php/reefd/article/view/644/573

Bracht, V. (2010). A educação física no ensino fundamental. En R. De Casia, L. Licíno \&A. F. Barbosa(Org.) ISeminário nacional do Currículo em Movimento. Secretaria de Educação Básica. Belo Horizonte (Brasil).

Cañabate, D., Tesouro, M., Puiggali, J., \& Zagalaz, M. L. (2019). Estado actual de la Educación Física desde el punto de vista del profesorado. Propuestas de mejora. RETOS. Nuevas Tendencias en Educación Física, (35), 47-53. Recuperado de: https://recyt.fecyt.es/index.php/ retos/article/view/63038/40398

Águila, C., \& López, J. J. (2019). Cuerpo, corporeidad y educación: una mirada reflexiva desde la Educación Física. RETOS. Nuevas Tendencias en Educación Física, (35), 413-21. Recuperado de: https://recyt.fecyt.es/index.php/ retos/article/view/62035/41748

Centro de Estudos e Pesquisas em Educação, Cultura e Ação Comunitária. (2007). Guia de políticas de Educação Integral: orientações para implementação no municipio. São Paulo, Brasil: CENPEC / UNICEF. Recuperado de: https://sinapse.gife.org.br/download/guia-politicas-deeducacao-integral-orientacoes-para-implementacao-nomunicipio

Coe, D. P., Pivarnik, J. M., Womack, C. J., Reeves, M. J., \& Malina, R. M. (2012). Health-related fitness and academic achievement in middle school students. The Journal of Sports Medicine and Physical Fitness, 52(6), 654-60. Recuperado de: https://www.minervamedica.it/en/ j ournals / s p ort s - med - phy s i cal - fit nes s / article.php?cod=R40Y2012N06A0654\&acquista=1

De los Mozos \& Touya, I. (2014). La Ley Orgánica para la Mejora de la Calidad Educativa: una reforma contestada, pero de corto alcance. Revista española de pedagogía, 72(257), 23-37. Recuperado de: https:// revistadepedagogia.org/wp-content/uploads/2014/02/ 257-02.pdf

Ericsson, I., \& Karlsson, M. K. (2014). Motor skills and school performance in children with daily physical education in school - a 9-year intervention study: Daily physical activity in school. Scandinavian Journal of Medicine \& Science in Sports, 24(2), 273-8. https://doi.org/10.1111/j.16000838.2012.01458.x

Eurydice (2013). La educación física y el deporte en los centros escolares de Europa. Informe de Eurydice. Luxemburgo: Oficina de Publicaciones de la Unión Europea. Recuperado de: https://sede.educacion.gob.es/ publiventa/descarga.action?f_codigo_agc=16113

Franco, E., Coterón, J., Martínez, H. A., \& Brito, J. (2017). Perfiles motivacionales en estudiantes de educación física de tres países y su relación con la actividad física. Suma Psicológica, 24(1), 1-8. https://doi.org/10.1016/ j.sumpsi.2016.07.001

Gallego-Ortega, J. L., \& Rodríguez-Fuentes, A. (2018) Percepciones del profesorado sobre competencias comunicativas de futuros maestros de educación física.
Revista Internacional de Medicina y Ciencias de la Actividad Física y el Deporte, 18(71), 479-92. https:// .doi.org/10.15366/rimcafd2018.71.005

Haapala, E. A. (2013). Cardiorespiratory fitness and motor skills in relation to cognition and academic performance in children. Areview. Journal of Human Kinetics, 36, 5568. https://doi.org/10.2478/hukin-2013-0006

International Society for Physical Activity and Health. (2017). The Bangkok Declaration on Physical Activity for Global Health and Sustainable Development. British Journal of Sports Medicine, 51(19), 1389-91. https://doi.org/10.1136/ bjsports-2017-098063

López-Gil, J. F. (2018). Competencias clave en educación primaria: relación con la Educación Física dentro de la región de Murcia. EmásF: revista digital de educación física, (55), 107-22. Recuperado de: https:// dialnet.unirioja.es/servlet/articulo?codigo $=6702837$

Ministério do Esporte. (2013). Diagnóstico Nacional do Esporte. Brasilia, Brasil: Ministério do Esporte. Recuperado de: http://www.esporte.gov.br/diesporte/

Ministério da Educação. (1996). Lei de Diretrizes e Bases da Educação Nacional, de 20 de dezembro. Brasilia, Brasil: Diário Oficial. Recuperado de: http://www.planalto.gov.br/ ccivil_03/leis/19394.htm

Ministério da Educação. (1997). Parâmetros curriculares nacionais: introduc'aमo aos parametros curriculares nacionais. Brasília, Brasil: MEC/SEF. Recuperado de: http://portal.mec.gov.br/seb/arquivos/pdf/livro01.pdf

Ministerio de Educación y Ciencia. (2006). Ley Orgaìnica 2/ 2006, de 3 de mayo, de Educacioìn. Madrid, España: Boletín Oficial del Estado. Recuperado de: https:// www.boe.es/buscar/pdf/2006/BOE-A-2006-7899consolidado.pdf

Ministerio de Educación y Ciencia. (2007). Orden ECI/3857/ 2007, de 27 de diciembre, por la que se establecen los requisitos para la verificación de los títulos universitarios oficiales que habiliten para el ejercicio de la profesión de Maestro en Educación Primaria. Madrid, España: Boletín Oficial del Estado. Recuperado de: https:// www.boe.es/boe/dias/2007/12/29/pdfs/A53747-53750.pdf Ministério da Educação. (2009). Rede de saberes mais educação: pressupostos para projetos pedagógicos de educação integral: caderno para professores e diretores de escolas. Série Mais Educação. Brasília, Brasil: MEC. Recuperado: http://portal.mec.gov.br/dmdocuments/ cad_mais_educacao_2.pdf

Ministério da Educação. (2010). Diretrizes Curriculares Nacionais. Brasília, Brasil: MEC. Recuperado de: http://

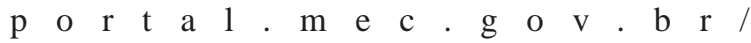
index.php?option=com_docman\&view=download\&alias=15548d-c-n-educacao-basica-nova-pdf\&Itemid=30192

Ministério da Educação. (2013). Programa Mais Educação: passo a passo. Série Mais Educação. Brasília, Brasil: MEC. Recuperado de: http://portal.mec.gov.br/ dmdocuments/passoapasso_maiseducacao.pdf

Ministério da Educação. (2017). Base Nacional Comum Curricular. Brasília, Brasil: MEC. Recuperado de: http:// basenacionalcomum.mec.gov.br/images/ BNCC_EI_EF_110518_versaofinal_site.pdf

Ministerio de Educación, Cultura y Deporte. (2014). Real 
Decreto 126/2014, de 28 de febrero, que establece el currículo básico de la Educación Primaria. Madrid, España: Boletín Oficial del Estado. Recuperado de: https:/ /www.boe.es/buscar/pdf/2014/BOE-A-2014-2222consolidado.pdf

Ministerio de Educación, Cultura y Deporte. (2013). Ley Orgánica 8/2013, de 9 de diciembre, para la Mejora de la Calidad Educativa. Madrid, España: Boletín Oficial del Estado. Recuperado de: https://www.boe.es/buscar/pdf/ 2013/BOE-A-2013-12886-consolidado.pdf

Méndez, D., Fernández-Río, J., Méndez, A., \& Prieto, J. (2015). Análisis de los currículos autonómicos LOMCE de Educación Física en Educación Primaria. RETOS. Nuevas Tendencias en Educación Física, Deporte y Recreación, (28), 15-20. Recuperado de: https://recyt.fecyt.es/ index.php/retos/article/view/34822/19057

Méndez, D., Pérez, A. Méndez, A., Fernández, F. J., \& Prieto, J.A. (2017). Análisis del desarrollo curricular de la Educación Física en la Enseñanza Secundaria Obligatoria: Comparación de los currículos autonómicos. RETOS. Nuevas Tendencias en Educación Física, (31), 82-7. Recuperado de: https://recyt.fecyt.es/index.php/retos/article/ view/42577/31468

Moll, J. (2012). Caminhos da Educação Integral no Brasil: direito a outros tempos e espaços educativos. Porto Alegre, Brasil: Penso.

Muñoz, J. C. (2014). El currículo de Educación Física en educación primaria en la LOMCE. EmásF: Revista digital de Educación Física, (27), 24-39. Recuperado de: https:// dialnet.unirioja.es/descarga/articulo/4736174.pdf

Nunes, H. C., \& Neira, M. G. (2017). A diferença no currículo cultural: por uma Educação (Física) menor. Praxis Educativa, 12(2), 464-80. https://doi.org/10.5212/ PraxEduc.v.12i2.0010

Organización para la Cooperación y el Desarrollo Económicos. (2016). PISA 2015 assessment and analytical framework: science, reading, mathematic, financial literacy and collaborative problem solving (Revised edition). París, Francia: OECD. Recuperado de: https:// read.oecd-ilibrary.org/education/pisa-2015-assessmentand-analytical-framework_9789264281820-en\#page1

Organización Mundial de la Salud. (2014). Recomendaciones mundiales sobre actividad física para la salud. Ginebra, Suiza: OMS. Recuperado de: https://apps.who.int/ i r is / bi t s trea m/hand le/10665/44441/

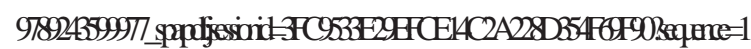

Organización Mundial de la Salud. (2017). Actividad física para la salud: más personas activas para un mundo más sano: proyecto de plan de acción mundial sobre actividad física 2018-2030: informe del Director General (No. EB142/18). Lisboa, Portugal: OMS. Recuperado de: https://apps.who.int/iris/bitstream/handle/ $10665 / 274122 /$ B 142 - 18 sp.pdf?sequence $=1$ \&isAllowed $=\mathrm{y}$

Organización Mundial de la Salud. (2018a). Actividad física. Datos y cifras. Recuperado de: https://www.who.int/es/ news-room/fact-sheets/detail/physical-activity

Organización Mundial de la Salud. (2018b). Plan de accioìn mundial de la OMS sobre actividad fiìsica 2018-2030. Ginebra, Suiza: OMS. Recuperado de: https:// apps.who.int/iris/bitstream/handle/10665/274570/
B142_R5-sp.pdf?sequence $=1$ \&isAllowed=y

Physical Activity Guidelines Advisory Committee (2018). Physical Activity Guidelines Advisory Committee Scientific Report, 2018. Washington, EE.UU.: US Department of Health and Human Services. Recuperado de: https://health.gov/paguidelines/second-edition/ report/pdf/pag_advisory_committee_report.pdf

Sallis, J. F., Bull, F., Guthold, R., Heath, G. W., Inoue, S., Kelly, P., ..., \& Hallal, P. C. (2016). Progress in physical activity over the Olympic quadrennium. The Lancet, 388(10051), 1325-36. https://doi.org/10.1016/S0140-6736(16)30581-5

Sallis, J. F., McKenzie, T. L., Beets, M. W, Beighle, A., Erwin, H., \& Lee, S. (2012). Physical Education's role in public health: Steps forward and backward over 20 years and HOPE for the future. Research Quarterly for Exercise \& Sport, 83(2), 125-35. http://doi.org/10.1080/ 02701367.2012.10599842

Saviani, D. (2010). Sistema Nacional de Educação articulado ao Plano Nacional de Educação. Revista Brasileira de Educação, 15(44). Recuperado de: http://www.scielo.br/ pdf/rbedu/v15n44/v15n44a13.pdf

Taylor, I. M., Spray, C., \& Pearson, N. (2014). The influence of the physical education environment on children's wellbeing and physical activity across the transition from primary to secondary school. Journal of Sport and Exercise Phychology, 36(6), 574-83. http://doi.org/ 10.1123/jsep. 2014-0038

Torrijos-Niño, C., Martínez-Vizcaíno, V., Pardo-Guijarro, M. J., García-Prieto, J. C., Arias-Palencia, N. M., \& SánchezLópez, M. (2014). Physical fitness, obesity, and academic achievement in schoolchildren. The Journal of Pediatrics, 165(1), 104-9. https://doi.org/10.1016/j.jpeds.2014.02.041

Trudeau, F., \& Shephard, R. J. (2008). Physical education, school physical activity, school sports and academic performance. International Journal of Behavioral Nutrition and Physical Activity, 5(1), 10. https://doi.org/ 10.1186/1479-5868-5-10

Organización de las Naciones Unidas para la Educación, la Ciencia y la Cultura. (1979). Actas de la conferencia general: 20a reunión, París, 24 de octubre-28 de noviembre de 1978. París, Francia: UNESCO. Recuperado de: https://unesdoc.unesco.org/ark:/ 48223 / pf0000114032_spa

Organización de las Naciones Unidas para la Educación, la Ciencia y la Cultura. (2015). Educación física de calidad (EFC): Guía para los responsables políticos. París, Francia: UNESCO. Recuperado de: https:// unesdoc.unesco.org/ark:/48223/pf0000231340

Organización de las Naciones Unidas para la Educación, la Ciencia y la Cultura. (2017). Sexta conferencia internacional de ministros y altos funcionarios encargados de la educacioìn fiìsica y el deporte: Plan de acción de Kazán (SHS/2017/5 REV). París, Francia: UNESCO. Recuperado de: https://unesdoc.unesco.org/ark:/48223/ pf0000252725_spa

Warburton, D. E., Charlesworth, S., Ivey, A., Nettlefold, L., \& Bredin, S. S. (2010). A systematic review of the evidence for Canada's Physical Activity Guidelines for Adults. International Journal of Behavioral Nutrition and Physical Activity, 7(1), 39. https://doi.org/10.1186/14795868-7-39 